\title{
Effect of Varying the Intensity and Train Frequency of Forelimb and Cerebellar Mossy Fiber Conditioned Stimuli on the Latency of Conditioned Eye-Blink Responses in Decerebrate Ferrets
}

\author{
Pär Svensson, ${ }^{1}$ Magnus Ivarsson, and Germund Hesslow \\ Department of Physiology and Neuroscience \\ Lund University \\ S-223 62 Lund, Sweden
}

\begin{abstract}
To study the role of the mossy fiber afferents to the cerebellum in classical eye-blink conditioning, in particular the timing of the conditioned responses, we compared the effects of varying a peripheral conditioned stimulus with the effects of corresponding variations of direct stimulation of the mossy fibers. In one set of experiments, decerebrate ferrets were trained in a Pavlovian eye-blink conditioning paradigm with electrical forelimb train stimulation as conditioned stimulus and electrical periorbital stimulation as the unconditioned stimulus. When stable conditioning had been achieved, the effect of increasing the intensity or frequency of the forelimb stimulation was tested. By increasing the intensity from 1 to $2 \mathrm{~mA}$, or the train frequency from 50 to $100 \mathrm{~Hz}$, an immediate decrease was induced in both the onset latency and the latency to peak of the conditioned response. If the conditioned stimulus intensity/frequency was maintained at the higher level, the response latencies gradually returned to preshift values. In a second set of experiments, the forelimb stimulation was replaced by direct train stimulation of the middle cerebellar peduncle as conditioned stimulus. Varying the frequency of the stimulus train between
\end{abstract}

\footnotetext{
${ }^{1}$ Corresponding author.
}

50 and $100 \mathrm{~Hz}$ had effects that were almost identical to those obtained when using a forelimb conditioned stimulus. The functional meaning of the latency effect is discussed. It is also suggested that the results support the view that the conditioned stimulus is transmitted through the mossy fibers and that the mechanism for timing the conditioned response is situated in the cerebellum.

\section{Introduction}

An important feature of the classical or Pavlovian learning paradigm is the precise temporal regulation of the conditioned response (CR). In classical eye-blink conditioning, where an animal has learned to blink in response to a conditioned stimulus (CS), such as a tone or electrical skin stimulation, the CR is timed so that the eye is maximally closed and, hence, under natural circumstances, protected at the time of the unconditioned stimulus (US), usually an air puff or a periorbital electrical stimulus. This holds over a wide variety of experimental situations. If the interval between the CS and the US is changed, the animal will learn to change the latency of the CR, so that its protective effect is restored (for references, see Mackintosh 1974). If rabbits are trained with two different tone frequencies as CSs, each tone being followed by the US at a different interval, the animals will learn to emit two CRs with latencies appropriate to each CS (Mauk and Ruiz 1992).

An exception to this rule is the shift in CR onset latency that occurs when the animals have

LEARNING \& MEMORY 3:105-115 C 1997 by Cold Spring Harbor Laboratory Press ISSN1072-0502/97 \$5.00

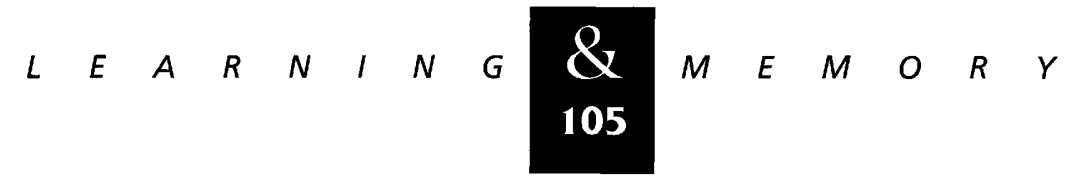


been trained to a particular CS and the intensity or the frequency of this CS is changed. For instance, Pavlov reported that if the frequency of a CS consisting of rhythmic tactile stimulation was increased, a conditioned salivation response appeared earlier (Pavlov 1927, p. 100). More recently, it has been shown that varying the intensity of a tone CS can affect the latency of a conditioned nictitating membrane response (Frey 1969; Leonard and Monteau 1971). A limitation of these studies is that they could not exclude that the latency effects were merely secondary to increases in the CR amplitude. A gradual change of the CS intensity during an individual trial has also been shown to change the CR latency. A gradual increase from, as well as a decrease to, the training intensity caused shorter CR latencies (Kehoe et al. 1995).

The aim of the present study was to determine whether the effect on CR latency of changing the intensity of a peripheral CS could be mimicked when using direct stimulation of the mossy fibers to the cerebellum as the CS. Several lines of converging evidence now suggest that the cerebellum plays a key role in the learning as well as the performance of classically conditioned somatic responses. For instance, a large number of studies have shown that lesions to the anterior interpositus nucleus and of the cerebellar cortex (for review, see Yeo 1991; Thompson and Krupa 1994), as well as pharmacological (Krupa et al. 1993) or physiological (Hesslow 1994) inactivation severely interfere with eye-blink conditioning. Several investigators have suggested that conditioning might utilize mechanisms that have been proposed previously by Brindley (1964), Marr (1969), and Albus (1971) for other forms of motor learning, namely that climbing fibers to the cerebellum can modify the responsiveness of Purkinje cells to mossy fiber/ parallel fiber input (for review, see Ito 1984). In the context of classical conditioning this would mean that the CS activates mossy fibers and the US climbing fibers (cf. Fig 1). Several experimental results support this suggestion. For instance, it has been shown that stimulation of the pontine nuclei, the source of a large group of mossy fibers, which enter the cerebellum via the middle cerebellar peduncle (MCP), can be used as CS (Steinmetz et al 1989; Steinmetz 1990).

Although the evidence is very strong that the cerebellum is important for classical conditioning, it is still not clear precisely which aspects of conditioning are controlled by the cerebellum. It is possible that the CS information undergoes im-

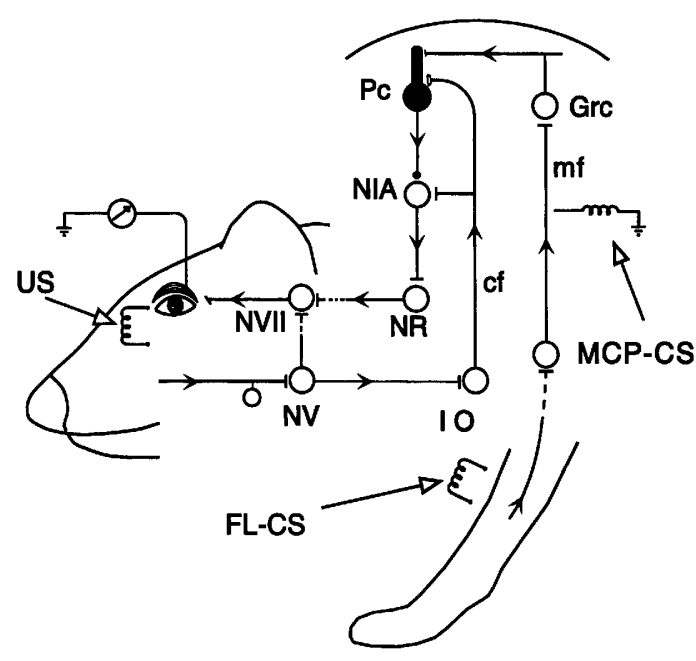

Figure 1: Experimental setup with hypothetical wiring diagram. Conditioned stimulation was applied to a forelimb (FL-CS) or to the middle cerebellar peduncle (MCP_CS). The unconditioned stimulus (US) was applied to the periorbital area. The CR was recorded as EMG activity from the upper eyelid. The hypothetical pathway for the CS is from the forelimb via mossy fibers $(\mathrm{mf})$, granule cells (Grc), to the Purkinje cell (Pc). The hypothetical pathway for the US from the periorbital area is via the trigeminal nucleus (NV), the inferior olive (IO), and climbing fibers (cf) to Pc. Output is from the cerebellar cortex to the anterior interpositus nucleus (NIA), red nucleus (NR), and facial nucleus (NVII) to the orbicularis oculi muscle.

portant processing in the brain stem or in forebrain structures before it reaches the cerebellum. For instance, several investigators have suggested that the hippocampus performs a temporal processing that is utilized by the cerebellum (see e.g., Port et al. 1986; Christiansen and Schmajuk 1992). It is therefore of interest to determine to what extent conditioning, which uses direct stimulation of the mossy fiber input to the cerebellum as the CS, resembles conditioning to a peripheral CS.

In the first part of the study, we investigated the effects on CR latency of changing the current intensity or pulse frequency of a CS consisting of a train of electrical stimuli to the forelimb. In the second part, we studied the effects of changing the frequency of the CS when using direct stimulation of mossy fibers in the MCP as the CS. If the effects of varying the intensity or train frequency of a peripheral CS can be mimicked by variations in the frequency of direct mossy fiber stimulation, this would support the hypothesis that

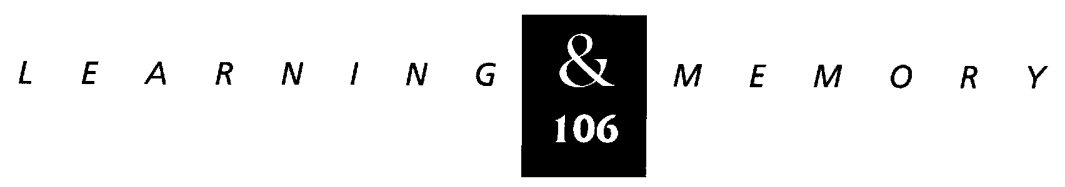


conditioning occurs in the cerebellum and that the CS is transmitted via mossy fibers. It would also indicate that the essential parts of the timing mechanisms reside in the cerebellum.

\section{Materials and Methods}

\section{ANESTHESIA AND SURGERY}

The experiments were performed on 23 male ferrets $(0.8-1.8 \mathrm{~kg})$. The animals were deeply anesthetised with isoflurane (Abbot Laboratories Ltd., UK, $1.5 \%-2 \%$ in a mixture of $\mathrm{O}_{2}$ and $\mathrm{N}_{2} \mathrm{O}$ ). They were initially placed in a box into which anesthetic gas was directed. When deep anesthesia had been achieved, a tracheotomy was performed and the gas was then channeled directly into a tracheal tube. The level of anesthesia was monitored regularly by testing withdrawal reflexes.

The animal's head was fixed to a stereotaxic frame. The skull was opened on the left side, and the caudal half of the right and the left cerebral hemispheres and a substantial part of the thalamus on the left side were removed by aspiration. The aspiration exposed the left lateral part of the cerebellum and the superior and the inferior colliculus of the brain stem. The animal was then decerebrated by a section with a blunt spatula through the brain stem $0.5-1 \mathrm{~mm}$ rostral to the superior colliculus and the red nucleus. The completeness of the decerebration was verified in all cases by postmortem examination. In those animals in which stimulation of MCP was tested, the peduncle was exposed on the left side by removing the most lateral part of the tentorium. This procedure also exposed a small portion of the trigeminal nerve. To further expose the MCP, in four animals, the most lateral part of the inferior colliculus was aspirated. Bleeding was controlled with gelfoam. After decerebration the anesthesia was terminated. The end-expiratory $\mathrm{CO}_{2}$ concentration, arterial blood pressure, and the rectal temperature were monitored continuously and kept within physiological limits. Throughout the experiment the animals received artificial ventilation and a continuous intravenous infusion $(50 \mathrm{mg} / \mathrm{ml}$ of glucose, isotonic acetate Ringer's solution, $60 \mathrm{mg} /$ ml of Macrodex with $\mathrm{NaCl}$; proportion $1: 1: 1 ; 1$ $\mathrm{ml} / \mathrm{kg}$ per hour).

\section{TRAINING PROCEDURES}

All of the animals were first trained with a CS consisting of a 300-msec train of electrical stimuli to the left forelimb (FL-CS; $0.2 \mathrm{msec}$ square wave pulses, $50 \mathrm{~Hz}, 1 \mathrm{~mA}$ ) applied through two subcutaneous needle electrodes, one placed laterally and the other medially on the left upper forelimb. Later in the experiments, changes were introduced in the CS parameters, which are described in Results. In six animals the forelimb CS was replaced during the experiment by direct stimulation of mossy fibers using a tungsten wire electrode (diameter and length of deinsulated tip were 50 and $75 \mu \mathrm{m}$, respectively) inserted into the MCP (MCP-CS; 0.1 msec square wave pulses; other parameters are described in Results). The MCP had been exposed during the surgery, and the electrode could be positioned under visual guidance on the surface of the peduncle where it enters the cerebellum. The electrode was then lowered vertically $0.5-1 \mathrm{~mm}$. The depth of the electrode tip was adjusted so that CRs could be reliably elicited by MCP stimulation. The strength required for this varied among animals $(18-100 \mu \mathrm{A})$. If the electrode was lowered too deep (1-3 mm), the trigeminal nerve was activated. As shown in Figure 6D (below), single-pulse stimulation of the trigeminal nerve at $10 \mu \mathrm{A}$ elicited short latency eyelid electromyograph (EMG) responses that were very similar in latency to responses elicited by the US ( $\sim 4 \mathrm{msec})$. The stimulation site in the MCP was always verified by histological examination. The formaline-perfused cerebellum was sectioned and stained with Cresyl violet.

The US consisted of periorbital electrical stimulation through two stainless steel electrodes, the tips of which were inserted into the skin of the medial part of the periorbital area, $-5 \mathrm{~mm}$ apart. Three square pulses of $0.5 \mathrm{msec}$ duration $(50 \mathrm{~Hz})$ were used. The stimulus strength was $3 \mathrm{~mA}$. The excitability of the eye-blink reflex pathway was tested in naive animals or during adaptation by stimulating the periorbital area with a low-intensity US (one pulse of $0.5 \mathrm{msec}$ and $0.5-1 \mathrm{~mA}$ ) without a preceding CS.

The US was applied at the termination of the $\mathrm{CS}$, so the interstimulus interval (ISI) was 300 msec. CRs were obtained in all animals. In previous work with this preparation in our laboratory, neither sensitization nor pseudoconditioning has ever been observed (Hesslow and Ivarsson 1996; Ivarsson et al. 1997).

The intertrial interval (ITI) was kept constant at $20 \mathrm{sec}$ throughout most of the experiment. To exclude the possibility that the responses acquired

$$
\text { ……‥ 圈"no. }
$$


during training were attributable to temporal conditioning, the ITI was occasionally increased to 30$60 \mathrm{sec}$ in a pseudorandom manner over at least 15 trials. The acquired CRs always remained timelocked to the CS. This test was performed in every animal.

The eye-blink responses were monitored by EMG recordings from the orbicularis oculi muscles through two stainless steel electrodes inserted into the eyelid, $\sim 3-5 \mathrm{~mm}$ above the lateral margin of the left eye. Electrophysiological recordings and analysis were performed with computer software developed in our laboratory. The sampling rate was $5 \mathrm{kHz}$. To reduce the baseline shift caused by the US, the recordings were run through a digital high-pass filter before analysis began.

CALCULATIONS OF CR MAGNITUDE, UR MAGNITUDE, AND LATENCY TO CR ONSET AND PEAK

To analyze CR magnitude, UR magnitude, and CR latency, the EMG signal was rectified. The CR magnitude was defined as the integrated EMG activity from 60 to $299 \mathrm{msec}$ (in CS-US trials) or to $700 \mathrm{msec}$ (in CS-alone trials) after CS onset. The UR magnitude was the integrated EMG activity from 4 to 22 msec after US onset. The UR was analyzed only in trials where the US was a single pulse.

To standardize the measurements of onset and peak latency the EMG activity was integrated over 5-msec bins. The CR onset latency was taken as the start of the first 5-msec bin, of which the EMG activity exceeded the spontaneous activity during $100 \mathrm{msec}$ prior to CS onset by $200 \%$. The latency to peak was the latency to the start of the bin with the highest amplitude. An example of CR onset and peak in one trial is indicated in Figure 6B (below). To be classified as a CR the EMG response had to begin between $60 \mathrm{msec}$ and $299 \mathrm{msec}$ after the CS onset and have a CR peak amplitude of at least $200 \%$ of the spontaneous activity.

\section{Results}

\section{EFFECT OF VARYING THE FL-CS INTENSITY ON CR LATENCY}

Animals were trained until stable conditioning had been achieved. This took 5-8 hr (500-1000 trials) at which time CRs were elicited on at least 95\% of the trials. The onset latency of the CRs varied among the animals; the range of mean onset latencies was 99-248 msec. The latencies were relatively stable in each individual animal; the S.D. of the CR onset latency was $<30 \mathrm{msec}$ in 20 of the 23 animals, $35 \mathrm{msec}$ in two animals, and $41 \mathrm{msec}$ in one animal.

When the animals had acquired stable CRs, the CS intensity was shifted from the training level of 1 to $2 \mathrm{~mA}$. This caused an immediate shortening of the onset latency [observed in 23 of 23 animals tested; (23/23)]. The size of this effect varied among animals. The mean latency change was 59 msec, and the range was $15-107 \mathrm{msec}$. The onset latency remained at the shortened level until the CS was reset to the original $1 \mathrm{~mA}$ after 10-24 trials. Results from a representative experiment are shown in Figure 2A. A second test in 13 of these animals had the same effect.

Increasing the CS intensity also caused an increase in CR magnitude (Fig. 2C). The shortening of the onset latency might therefore simply be a reflection of an increased $C R$ amplitude. In that case, one would not expect any change in the latency to the peak amplitude of the CR. However, the latency to peak was also shortened by increasing CS intensity (20/23; Fig. 2B) by about the same amount as the onset latency. The mean change in latency to peak in the 20 animals varied between 24 and $96 \mathrm{msec}$ as compared to 17 and $107 \mathrm{msec}$ for the onset latency change. This point is illustrated further in Figure 2E, which shows representative examples of two smoothed averages of 10 rectified CRs each, elicited by 1- and 2-mA CSs, respectively. Both the onset latency and the latency to peak of the CR elicited by the stronger CS are shortened. The records were taken from CSalone trials to get a clear picture of the topography of the CRs. It is noteworthy that the increased magnitude of these CRs was mainly attributable to a longer duration.

The change in CR latency was immediate and did not require any training. This was illustrated by experiments in which the CS intensity was alternated from trial to trial between 1 and $2 \mathrm{~mA}$. In these experiments, the latencies also changed in a regular manner from trial to trial (11/11). An example is shown in Figure $2 \mathrm{~F}$, which shows records from five consecutive trials.

It is well known that when the ISI is changed, the latency to the peak of the CR no longer coincides with the onset of the US. After further train-

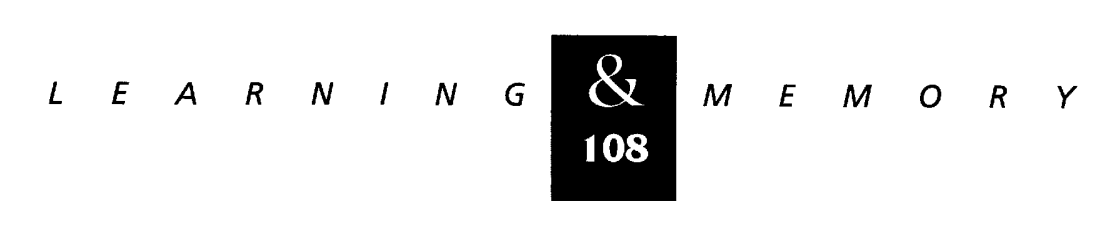



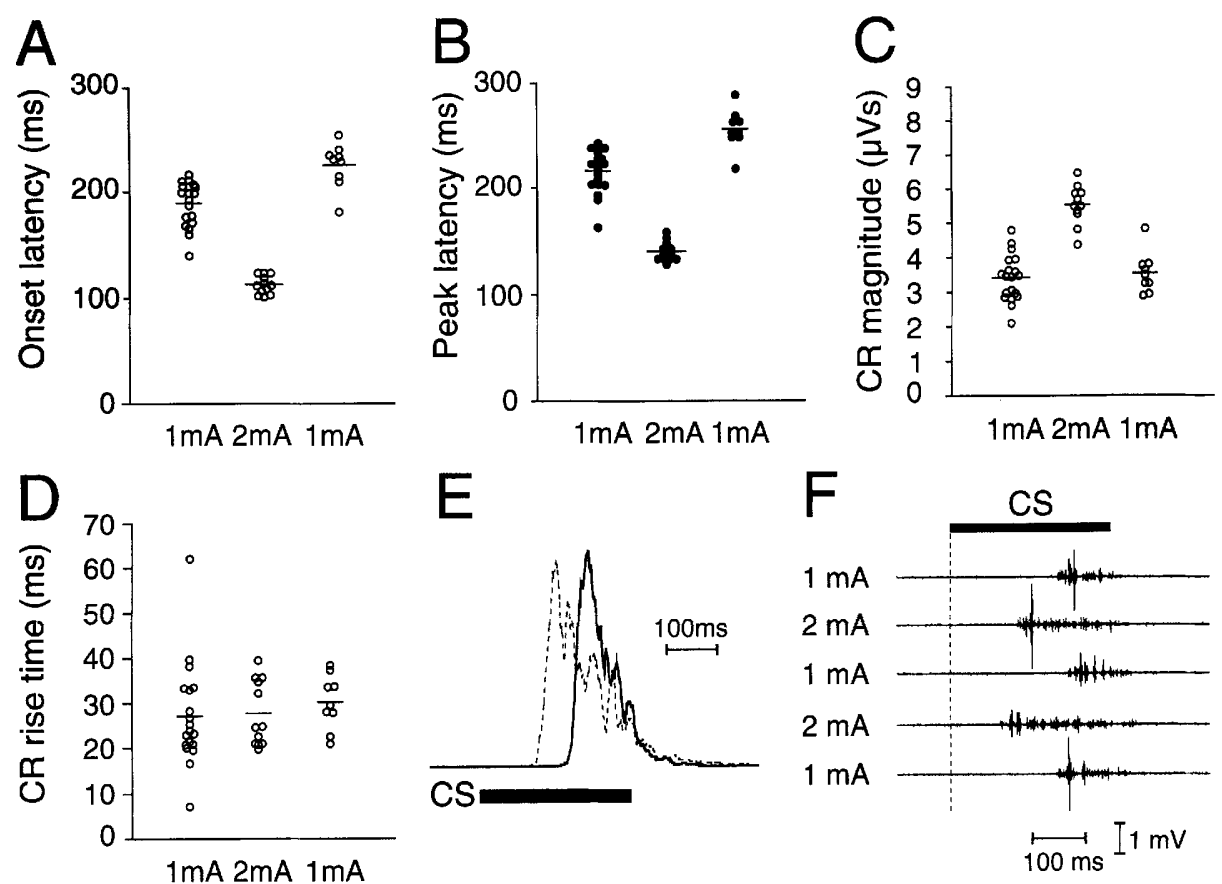

Figure 2: Effect of FL-CS intensity on the CR latency. The diagrams in $A-D$ are from the same animal and show the effects on onset latency $(A)$, latency to peak $(B), C R$ magnitude $(C)$, and CR rise time $(D)$ when the CS intensity was $1 \mathrm{~mA}$, increased to $2 \mathrm{~mA}$, and then reset to $1 \mathrm{~mA}$, respectively. Each circle corresponds to one trial, and the horizontal bar is the corresponding mean. The effect on CR topography is illustrated in $E$ by rectified and averaged EMG recordings from CS-alone trials with a stimulation intensity of first $1 \mathrm{~mA}$ (solid line, $n=10$ ) and then $2 \mathrm{~mA}$ (broken line, $n=11$ ). These records were smoothed by applying a running average of 19 values, corresponding to $3.8 \mathrm{msec}$. $(F)$ Five consecutive EMG records from the upper eyelid in another animal when the CS intensity was alternated from trial to trial.

ing the CR latency changes until the peak again coincides with the postshift US onset. Because increased CS intensity also causes a suboptimal CR (with latency of the $\mathrm{CR}$ too short to give maximal protection) it may be asked if this suboptimal CR, with further training, will return to the original latency. This suggestion is supported by an observation made in some experiments, namely that when the CS intensity was reset to the original 1 $\mathrm{mA}$, after 10 trials or more at high CS intensity, the CR latencies were actually slightly longer than during the first control period (6/10). An example can be seen in Figure 2, A and B. The slightly longer latency may indicate an adaptation to the high CS intensity.

Accordingly, adaptability of CR latency was tested in 10 animals that had previously shown a latency shift on increased CS intensity, by increasing the CS intensity from 1 to $2 \mathrm{~mA}$ and then maintaining it at $2 \mathrm{~mA}$. The increase in CS intensity from 1 to $2 \mathrm{~mA}$ immediately induced a decrease in CR onset latency and latency to peak, as described above. As the CS intensity was maintained at $2 \mathrm{~mA}$, however, both the onset and peak latencies were gradually prolonged and eventually returned to the control level. To estimate the rate of this adaptation, we divided the training period into five trial sessions and calculated the average latencies during each session. The number of sessions required until the onset latencies had increased to at least $95 \%$ of the average latency during a preceding control period varied between 4 and 22 (20 and 110 trials). We chose to illustrate this with plots from two extreme cases, with the one in Figure $3 \mathrm{~A}$ being an animal with fast adaptation, and in Figure 3B an animal with slow adaptation of the CR latency. A second and third test in one of these animals had the same effect. The results are summarized in Table 1.

\section{EFFECT OF VARYING FL-CS TRAIN FREQUENCY ON CR LATENCY}

When the intensity of natural skin stimulation is increased, the number of activated afferent fibers

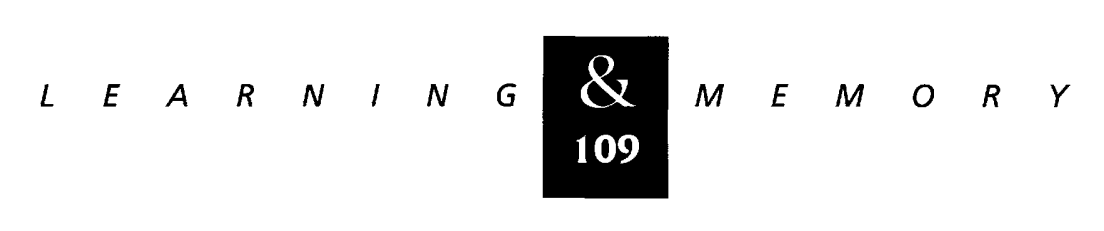



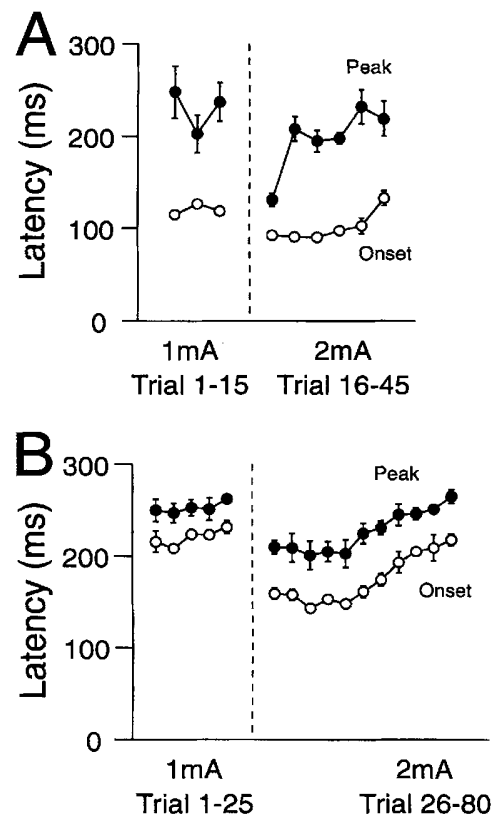

Figure 3: Adaptation of CR latency to high FL-CS intensity. When CS intensity was increased from 1 to 2 $\mathrm{mA}$, the CR onset latency $(O)$ and latency to peak (O) were immediately shortened. When the CS intensity was maintained at $2 \mathrm{~mA}$, the onset latency and the latency to peak gradually returned to control levels. (A) A case with a fast return; $(B)$ a case with a slow return to control latency. Each data point represents the mean \pm S.E.M of five consecutive trials.

and also the impulse frequency in these fibers will usually increase. As increasing the intensity of electrical skin stimulation decreases CR latency, it may be expected that changing the frequency of such stimulation would be comparable to changing the intensity and hence have the same effect on CR latency. Previous studies of the effect of CS frequency on the latency of eye-blink CRs have only used tone as the CS. But changing the frequency of the tone $\mathrm{CS}$ is not comparable to changing the intensity and cannot be compared to varying the frequency of electrical skin stimulation. Different tones are coded in the cochlea by different, although overlapping, populations of hair cells and may be viewed as different CSs. Changes in CS frequency can then be interpreted as a test of generalization between two closely related CSs. This is indicated by experiments where test trials with increased CS tone frequency decreased the likelihood of a CR but not the CR latency (Kehoe and Napier 1991).

We tested the effect of increasing the frequency of the CS train from 50 to $100 \mathrm{~Hz}$ in 13 animals. The effect was very similar to that of increasing the CS intensity (Fig. 4). Both the CR onset and peak latencies were shortened by increased CS train frequency $(13 / 13)$. The mean change of latency to onset was $67 \mathrm{msec}$ and varied between animals from 21 to $123 \mathrm{msec}$. The mean change in peak latency was $40 \mathrm{msec}$ and varied from 12 to 94 msec. The test was repeated in five of these animals and gave the same result. The effect of CS train frequency was also tested by alternating between high and low train frequencies from trial to trial. This resulted in immediate changes in CR latencies (12/12).

When the CS train frequency was maintained at $100 \mathrm{~Hz}$ in four animals, the CR latencies gradually returned to at least $95 \%$ of the preshift latency within 2-21 sessions (10-105 trials). This is illustrated with results from two animals in Figure 4, C and $\mathrm{D}$. A second and third test in one of these animals had the same effect. The results are summarized in Table 1.

\section{EFFECT OF VARYING FL-CS INTENSITY AND TRAIN FREQUENCY ON UNCONDITIONED RESPONSE MAGNITUDE}

It might be claimed that the decrease in CR latency was a result of an increase in the general excitability or arousal induced by the increased intensity, and not specific to the CS-CR pathway. To test this possibility, the effect of increasing CS intensity on eye-blink reflex excitability was studied in naive, nonconditioned animals. Three naive, nontrained animals received first US alone trials (one pulse of $1 \mathrm{msec} ; 0.5 \mathrm{~mA}$ ). Then the animals received paired CS-US stimulation but with a weak US (one pulse of $1 \mathrm{msec}$; $0.5 \mathrm{~mA}$ ).

The weak US elicited submaximal unconditioned responses (URs), smaller than those elicited by $3 \mathrm{~mA}$. Preceding the US with a weak $(1 \mathrm{~mA})$ or a strong ( $2 \mathrm{~mA}) \mathrm{CS}$ had no significant effect on the amplitude of the UR (Fig. 5A). This suggests that the CR latency decrease was not attributable to a general increase in excitability or arousal.

A similar test was performed to exclude that the adaptation of the CR latency to a permanent high-intensity CS was attributable to a change in excitability. Thus, UR magnitude was measured before, during, and after such adaptation, again with no systematic effects on excitability (Fig. 5B).

Equivalent tests of the effects of CS parameters on UR magnitude were performed in three animals

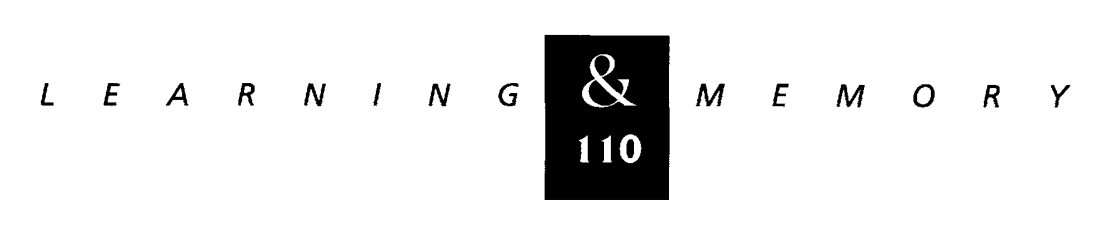


Table 1: Long-term adaptation of CR latency during high CS intensity or frequency

\begin{tabular}{|c|c|c|c|c|c|c|}
\hline \multirow[b]{3}{*}{ Animal no. } & \multicolumn{4}{|c|}{$C R$ latency ${ }^{a}$} & \multirow{2}{*}{\multicolumn{2}{|c|}{$\begin{array}{l}\text { Sessions } \\
\text { required for adaptation }\end{array}$}} \\
\hline & \multicolumn{2}{|c|}{ control } & \multicolumn{2}{|c|}{ first test session ${ }^{b}$} & & \\
\hline & onset & peak & onset & peak & onset & peak \\
\hline \multicolumn{7}{|c|}{ Forelimb CS, intensity } \\
\hline $1: 1$ & $106 \pm 7$ & $231 \pm 18$ & $93 \pm 2$ & $131 \pm 7$ & 5 & 5 \\
\hline $1: 2$ & $120 \pm 4$ & $229 \pm 14$ & $93 \pm 3$ & $131 \pm 7$ & 6 & 5 \\
\hline 2 & $195 \pm 10$ & $244 \pm 10$ & $99 \pm 6$ & $142 \pm 6$ & 8 & 6 \\
\hline 3 & $248 \pm 4$ & $273 \pm 4$ & $180 \pm 3$ & $226 \pm 2$ & 9 & 9 \\
\hline 4 & $99 \pm 1$ & $253 \pm 10$ & $84 \pm 17$ & $238 \pm 16$ & 10 & 2 \\
\hline 5 & $221 \pm 3$ & $252 \pm 3$ & $108 \pm 3$ & $210 \pm 5$ & 10 & 8 \\
\hline 6 & $225 \pm 3$ & $262 \pm 4$ & $139 \pm 6$ & $207 \pm 7$ & 11 & 10 \\
\hline 7 & $150 \pm 5$ & $245 \pm 9$ & $105 \pm 5$ & $172 \pm 16$ & 12 & 11 \\
\hline 8 & $109 \pm 4$ & $245 \pm 5$ & $72 \pm 3$ & $210 \pm 17$ & 14 & 4 \\
\hline 9 & $190 \pm 7$ & $257 \pm 6$ & $115 \pm 3$ & $205 \pm 11$ & 16 & 4 \\
\hline 10 & $203 \pm 9$ & $237 \pm 9$ & $121 \pm 7$ & $207 \pm 19$ & 22 & 10 \\
\hline \multicolumn{7}{|c|}{ Forelimb CS, frequency } \\
\hline $1: 1$ & $202 \pm 4$ & $261 \pm 6$ & $131 \pm 17$ & $229 \pm 9$ & 2 & 2 \\
\hline $1: 2$ & $200 \pm 9$ & $262 \pm 11$ & $137 \pm 25$ & $250 \pm 16$ & 2 & 2 \\
\hline $1: 3$ & $200 \pm 5$ & $253 \pm 12$ & $164 \pm 12$ & $232 \pm 10$ & 3 & 2 \\
\hline 2 & $163 \pm 7$ & $235 \pm 8$ & $114 \pm 10$ & $202 \pm 11$ & 5 & 2 \\
\hline 3 & $113 \pm 9$ & $210 \pm 10$ & $81 \pm 10$ & $183 \pm 48$ & 10 & 5 \\
\hline 4 & $250 \pm 5$ & $276 \pm 6$ & $127 \pm 3$ & $182 \pm 17$ & 21 & 15 \\
\hline \multicolumn{7}{|c|}{$M C P-C S$, frequency } \\
\hline 1 & $212 \pm 14$ & $256 \pm 7$ & $136 \pm 18$ & $173 \pm 9$ & 4 & 4 \\
\hline 2 & $107 \pm 9$ & $240 \pm 9$ & $59 \pm 2$ & $161 \pm 24$ & 5 & 4 \\
\hline $3: 1$ & $171 \pm 4$ & $269 \pm 3$ & $107 \pm 9$ & $228 \pm 7$ & 4 & 4 \\
\hline $3: 2$ & $195 \pm 5$ & $266 \pm 4$ & $91 \pm 12$ & $182 \pm 4$ & 9 & 7 \\
\hline
\end{tabular}

Note that three animals were tested more than once.

amsec, mean \pm S.E.M.

${ }^{\text {b } T e s t ~ s e s s i o n ~ w i t h ~ h i g h ~ i n t e n s i t y ~}(2 \mathrm{~mA})$ or high frequency $(100 \mathrm{~Hz})$. One session is five trials.

cNumber of sessions until the average session latency was at least $95 \%$ of control latency. One session is five trials.

by changing the frequency instead of the intensity of the CS. The results (not illustrated) were identical, that is, no significant effects were observed.

\section{EFFECT OF VARYING MCP-CS TRAIN FREQUENCY} ON CR LATENCY

In six of the animals that had been conditioned to a FL-CS, this CS was replaced by $50 \mathrm{~Hz}$ stimulation of the MCP. Figure $6 \mathrm{~A}$ shows the position of the stimulation electrode in a typical experiment. The records in $\mathrm{B}$ and $\mathrm{C}$ were obtained from trials with a FL-CS and MCP-CS respectively, in the same animal. The record in Figure 6D shows a short-latency response to a single-pulse stimulus when the electrode had been placed directly on the trigeminal nerve. The MCP-CS elicited CRs within $<10$ trials at stimulus strengths between 18 and $100 \mu \mathrm{A}$. The CRs elicited by MCP-CS were similar to those elicited by FL-CS with respect to both onset and peak latencies.

As shown for a representative case in Figure 7 , the effect of increasing the frequency of the MCP-CS train from 50 to $100 \mathrm{~Hz}$ was quite similar to that of changing the intensity or the frequency of a FL-CS. Both onset latency and latency to peak were decreased by the high-frequency

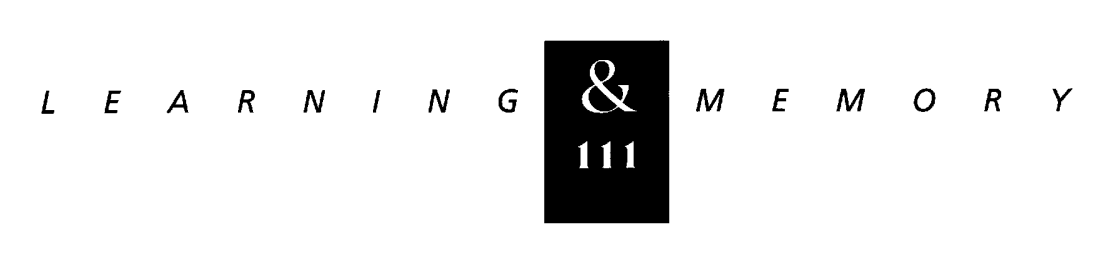



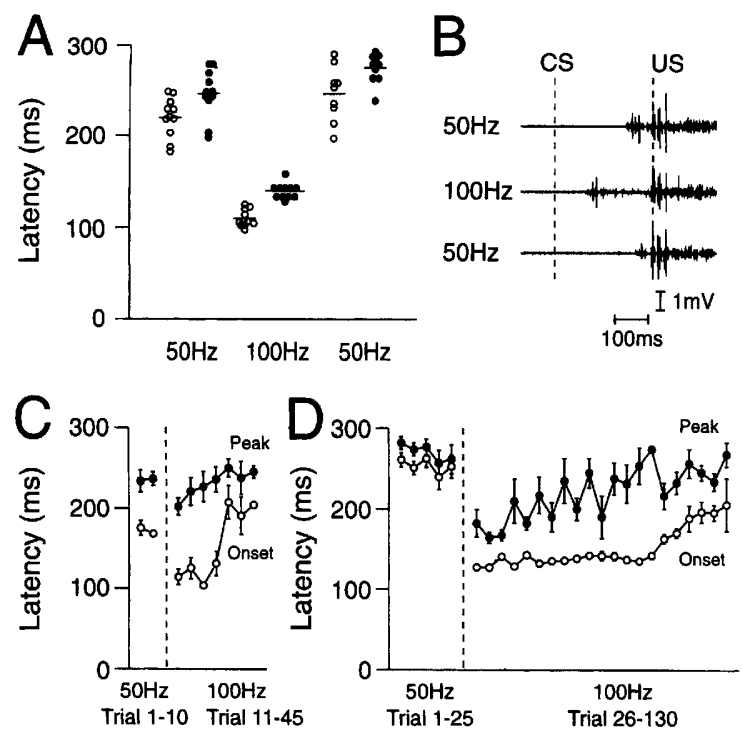

Figure 4: Effect of increasing the frequency of the $\mathrm{FL}-$ CS train on CR latency. The plot in $A$ shows CR onset latency $(O)$ and latency to peak $(O)$ when the CS frequency was $50 \mathrm{~Hz}$, increased to $100 \mathrm{~Hz}$, and reset to 50 $\mathrm{Hz}$ in a representative animal. (B) Sample records from each session in $A$. $C$ and $D$ show Adaptation of $C R$ latency when the train frequency of the $C S$ was maintained at $100 \mathrm{~Hz}$. (C) A case with a fast return; $(D)$ a case with a slow return to control latency.

MCP-CS in five out of five tested animals. Repeating the test a second time in four animals had the same effect. The mean change in onset latency varied from animal to animal between 8 and 145 msec and in latency to peak between 28 to 99 msec. Alternating between high and low frequency trains of stimuli to the MCP caused immediate changes in CR latency as described above for the FL-CS.

As shown above for FL-CS, when the higher MCP-CS train frequency was maintained, the CR onset latencies gradually adapted and returned to the pretest levels within four to nine sessions (2045 trials; Fig. 7C,D). This was observed in three out of three tested animals. A second test in one of these animals had the same effect. The results are summarized in Table 1.

\section{Discussion}

The main result of the first part of this study is the finding that an increase in the intensity of a FL-CS causes a reliable reduction of the CR la- tency. This effect was not just a reflection of an increased CR amplitude or strength, as suggested by Mackintosh (1974, p. 61), because the reduction in the latency to peak was similar. Because the change in CS intensity did not affect the magnitude of UR elicited with a weak US, it is also unlikely that the latency effects were attributable to a nonspecific increase in excitability, for instance, because of increased arousal.

Changes in the frequency of the forelimb CS had effects that were virtually indistinguishable from the intensity changes. Increasing the frequency from 50 to $100 \mathrm{~Hz}$ caused an immediate shortening of the CR latency. The similarity of these effects probably reflects a common mechanism. An increase in the frequency of the forelimb CS is likely to be translated into an increased firing frequency in the neurones of the afferent pathway. Although the high-intensity stimulus presumably
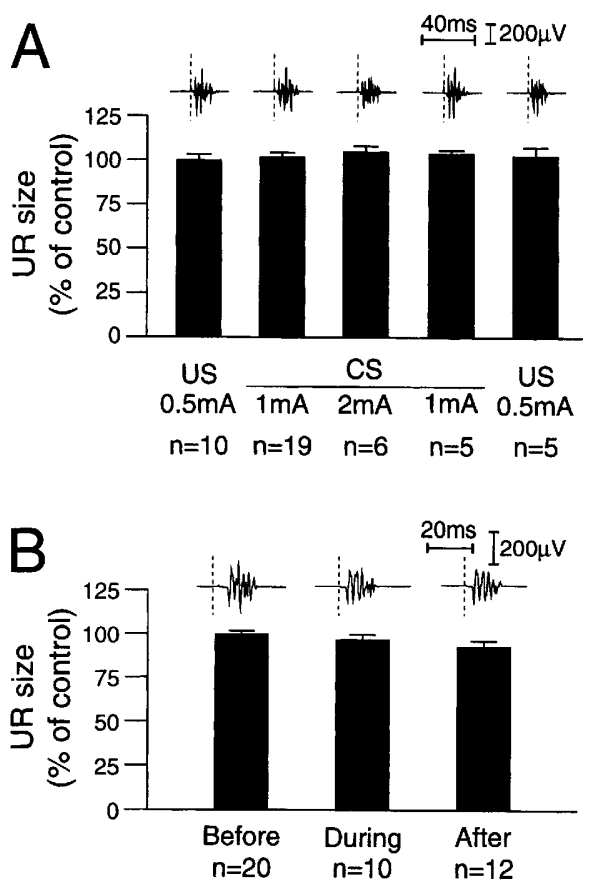

Figure 5: (A)Effect of CS intensity on UR magnitude in a naive subject. The first and fifth bars show the magnitude of URs elicited by a single $0.5-\mathrm{mA}$ periorbital stimulus in naive animals. Addition of a 1- or 2-mA CS (second and fourth bars) did not change the UR magnitude. $(B)$ Effect of maintaining high FL-CS intensity on UR magnitude. The CR magnitude was measured on US-alone ( 1 pulse, $0.5 \mathrm{~mA}$ ) trials before the CS intensity was increased (first bar), during adaptation to the higher intensity (second bar), and after the CR latencies had returned to the control level (third bar).

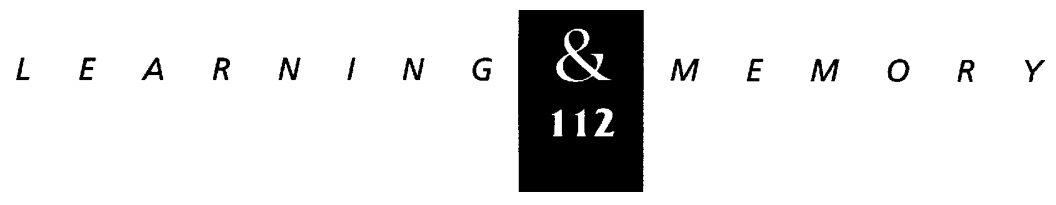



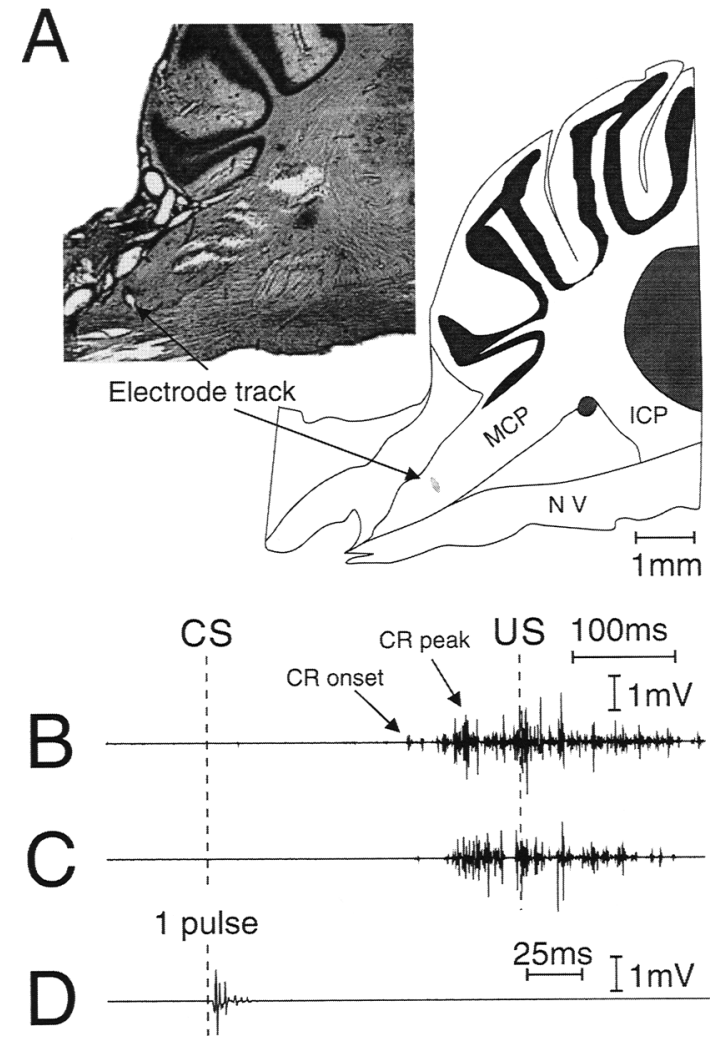

Figure 6: Placement of the electrode in the MCP in a representative experiment. $(A)$ Photograph and a reconstruction of a histological section. The electrode track in the MCP is indicated by an arrow. Also indicated are the trigeminal nerve (NV) and the inferior cerebellar peduncle (ICP). $B$ and $C$ show $C$ Rs elicited by FL-CS and $\mathrm{MCP}-\mathrm{CS}$, respectively in the same animal. The arrows in $B$ show the latencies to onset $(195 \mathrm{msec}$ ) and peak (255 msec) of the CR as determined by the computer software. When the electrode was too deep, direct activation of the trigeminal nerve by a single $10-\mu \mathrm{A}$ stimulus elicited a short-latency reflex response in the orbicularis oculi muscle $(D)$.

also recruited a larger population of afferent fibers in the forelimb, this might also be translated into a frequency code along the afferent pathways to the central locus of association.

It is generally assumed that the timing of the CR, such that the peak amplitude is reached at or just before the onset of the US, maximizes its protective effect. If so, the shortened latency that occurs when CS strength is increased might be thought to be dysfunctional. But there is another possibility. The intensity of a cause is often associated with a shorter temporal delay of the effect. The intensity of sounds, for instance, which may warn us against approaching predators or insects, usually indicate the distance to the sound source and therefore the time available for a protective response. Although this is a speculation, it would be wrong to dismiss the possibility that the latency effect described above is an important adaptive feature of classical conditioning.

The mechanism behind the timing of the CR is not known. A very simple hypothesis is that the CR latency reflects the time required for a temporal summation of postsynaptic potentials in modifiable synapses in the CS-CR pathway to reach firing threshold. A sudden increase in CS frequency or intensity would be expected to cause a reduction in the time required for reaching the threshold for eliciting a CR. This hypothesis is not new, and several theories and real time models have utilized similar ideas (cf. Desmond and Moore 1988; Grossberg and Schmajuk 1989).

We do not want to claim that this is the only mechanism governing $\mathrm{CR}$ timing. In fact, there is convincing evidence that some other timing mechanism must also be present. When the ISI is increased, the latency of the CR is also increased,
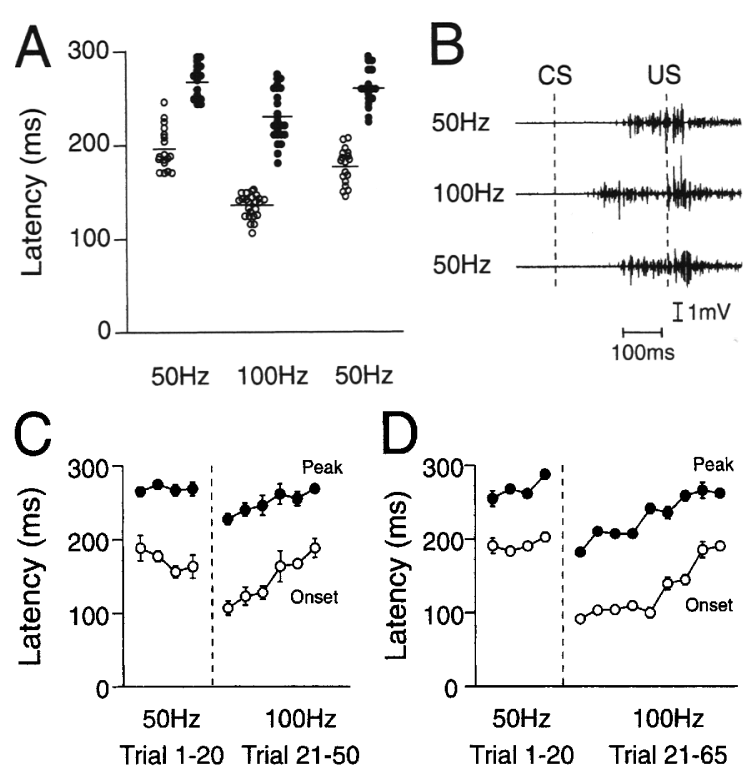

Figure 7: Effect of increasing the frequency of the MCP-CS train on CR latency. The plot in A shows CR onset latency $(O)$ and latency to peak $(O)$ when the CS frequency was $50 \mathrm{~Hz}$, increased to $100 \mathrm{~Hz}$, and reset to $50 \mathrm{~Hz}$ in a representative animal. (B) A sample record from each session in $A$. $C$ and $D$ show adaptation of $C R$ latency when the CS train frequency was maintained at $100 \mathrm{~Hz}$. (C) A case with a fast return; $(D)$ a case with a slow return to control latency.

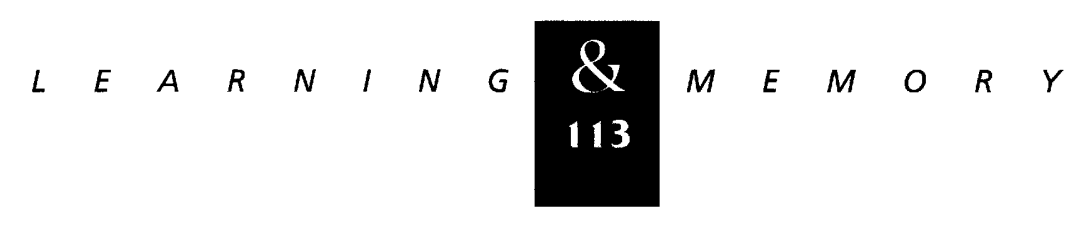


so that after a period of training, it again occurs just before the expected US. This change in latency can occur in two different ways. Sometimes the change has been described as a gradual shift of the CR onset latency toward the postshift US, with no concomitant change in CR probability (Coleman and Gormezano 1971; Norman et al. 1974). Other investigators have observed a disappearance of the preshift CR followed by the appearance of "new" CRs appropriately timed to the postshift US (Boneau 1958; Ebel and Prokasy 1963; Prokasy and Papsdorf 1965; Leonard and Theios 1967). This suggests that the timing is an integral part of what is learned when a CR is acquired. A CR would thus be learned to occur at a specific time. A change in CR latency to fit a new ISI would therefore require extinction of the inappropriately timed CR and acquisition of a new $C R$ with a latency appropriate to the new ISI.

If the timing of the CR was determined by temporal summation of postsynaptic potentials, changes in the size of these potentials would cause a gradual change in CR latency rather than extinction and reacquisition at a new latency. The observation of the present study - that the adaptation of the CR latency that occurs after a long-term increase in CS intensity involves a gradual sliding of the CR toward the new later US-fits well with the first description. This also supports the idea that there is a mechanism for CR timing that is independent of extinction and acquisition.

A possible conclusion is that there are at least two different timing mechanism. Perhaps the extinction and reacquisition mechanism accounts for adjustment to large ISI shifts, whereas the mechanism studied here can only operate on small time differences and therefore merely fine-tunes the CR latency. In the model suggested by Fiala et al. (1996) both mechanisms could be operative at the parallel fiber-Purkinje cell synapse.

The similarity between FL-CS and MCP-CS was striking. The CRs elicited by the two kinds of CS had surprisingly similar latencies, and the effects of varying the CS parameters were virtually identical. When the frequency of the MCP-CS train was increased, the effects were the same as those obtained when changing the parameters of a FLCS. The onset latency and the latency to peak of the CR were decreased by the increased MCP train frequency, and when the high frequency was maintained, the latencies returned to the original levels within a time span that was very close to that obtained with the FL-CS. This similarity of effect be- tween FL and MCP stimulation is highly suggestive of a similarity in mechanism.

As mentioned in the Introduction, previously it has been shown that stimulation of the pontine nuclei or mossy fibers can function as a CS. Such results support the hypothesis that the CS is transmitted to the cerebellum via the mossy fibers, but there are also other interpretations. Stimulation of pontine nuclei will probably activate fibers of passage. Mossy fiber stimulation will cause antidromic activation and may, via axon collaterals, inadvertently excite many other brain stem structures, one of which might be part of the actual CS pathway. The more closely direct mossy fiber stimulation can mimic a peripheral CS, together with converging evidence suggesting a key role for cerebellum in classical conditioning, the less likely such alternatives become; hence, the present findings suggest that the mossy fibers are the CS pathway.

Several investigators have suggested that although some of the essential conditioning mechanisms are located in the cerebellum, some processing of the CS information in forebrain structures, such as the hippocampus, is nevertheless required for the proper timing of the CR. The similarity in latency between CRs elicited by a FL-CS and a MCP-CS, as well as the similarity between the effects of varying FL-CS and MCP-CS parameters, suggests that the $C S$ information requires surprisingly little processing before entering the cerebellum. Although we cannot exclude that antidromic activation of the MCP has been important, our results suggest that the essential timing mechanisms are located in the cerebellum.

\section{Acknowledgments}

This study was supported by grants from the Medical Faculty, Lund University, the Swedish Medical Research Council (project no. 09899), and the Wallenberg foundation in cooperation with Astra AB.

The publication costs of this article were defrayed in part by payment of page charges. This article must therefore be hereby marked "advertisement" in accordance with 18 USC section 1734 solely to indicate this fact.

\section{References}

Albus, J.S. 1971. A theory of cerebellar function. Math. Biosci. 10: 25-61.

Boneau, C.A. 1958. The interstimulus interval and the latency of the conditioned eyelid response. J. Exp. Psychol. 56: 464-472.

Brindley, G.S. 1964. The use made by the cerebellum of the

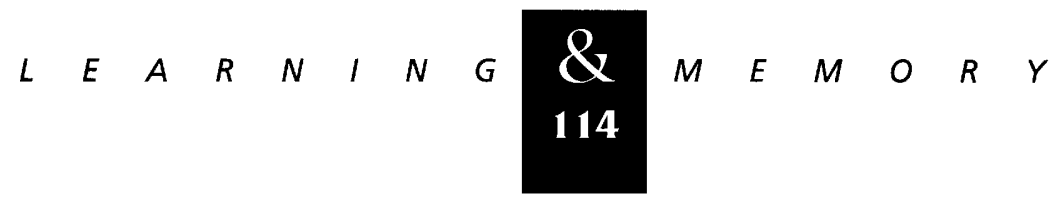


information that it receives from the sense organs. Int. Brain Res. Organ. Bull. 3: 80.

Christiansen, B.A. and N.A. Schmajuk. 1992.

Hippocampectomy disrupts the topography of the rat eyeblink response during acquisition and extinction of classical conditioning. Brain Res. 595: 206-214.

Coleman, S.R. and I. Gormezano. 1971. Classical conditioning of the rabbit's (Oryctolagus cuniculus) nictitating membrane response under symmetrical CS-US interval shifts. J. Comp. Physiol. Psychol. 77: 447-455.

Desmond, J.E. and J.W. Moore. 1988. Adaptive timing in neural networks: The conditioned response. Biol. Cybern. 58: 405-415.

Ebel, H.C. and W.F. Prokasy. 1963. Classical conditioning as a function of sustained and shifted interstimulus intervals. J. Exp. Psychol. 65: 52-58.

Fiala, J.C., S. Grossberg, and D. Bullock. 1996. Metabotropic glutamate receptor activation in cerebellar Purkinje cells as substrate for adaptive timing of the classically conditioned eye-blink response. J. Neurosci. 16: 3760-3774.

Frey, W.P. 1969. Within- and between-session CS intensity performance effects in rabbit eyelid conditioning. Psychon. Sci. 17: 1-2.

Grossberg, S. and N.A. Schmajuk. 1989. Neural dynamics of adaptive timing and temporal discrimination during associative learning. Neural Networks 2: 79-102.

Hesslow, G. 1994. Inhibition of classically conditioned eyeblink responses by stimulation of the cerebellar cortex in the cat. J. Physiol. 476: 245-225.

Hesslow, G. and M. Ivarsson. 1996. Inhibition of the inferior olive during conditioned responses in the decerebrate ferret. Exp. Brain Res. 110: 36-46.

Ito, M. 1984. The cerebellum and neuronal control. Raven Press, New York, NY.

Ivarsson, M., P. Svensson, and G. Hesslow. 1997. Bilateral disruption of conditioned response after unilateral blockade of cerebellar output in the decerebrate ferret. J. Physiol. 502: 189-201.

Kehoe, J.E. and R.M. Napier. 1991. Real-time factors in the rabbit's nictitating membrane response to pulsed and serial conditioned stimuli. Anim. Learn. Behav. 19: 195-206.

Kehoe, E.J., B.G. Schreurs, M. Macrae, and I. Gormezano. 1995. Effects of modulating tone frequency, intensity, and duration on classically conditioned rabbit nictitating membrane response. Psychobiology 23: 103-115.

Krupa, D.J., J.K. Thompson, and R.F. Thompson. 1993. Localization of a memory trace in the mammalian brain. Science 260: 989-991.
Leonard, D.W. and J.E. Monteau. 1971. Does CS intensity determine CR amplitude? Psychon. Sci. 23: 369-371.

Leonard, D.W. and J. Theios. 1967. Effect of CS-US interval shift on classical conditioning of nictitating membrane in the rabbit. J. Comp. Physiol. Psychol. 63: 355-358.

Mackintosh, N.J. 1974. The psychology of animal learning. Academic Press. London, UK.

Marr, D. 1969. A theory of cerebellar cortex. J. Physiol. 202: $437-470$.

Mauk, M.D. and B.P. Ruiz. 1992. Learning-dependent timing of pavlovian eyelid response: Differential conditioning using multiple interstimulus intervals. Behav. Neurosci.

106: 666-681.

Norman, R.J., J.R. Villablanca, K.A. Brown, J.A. Schwafel, and J.S. Buchwald. 1974. Classical eyeblink conditioning in the bilaterally hemispherectomized cat. Exp.Neurol. 44: 363-380.

Pavlov, I.P. 1927. Conditioned reflexes. (G.V. Anrep, Translation) Oxford University Press, London, UK.

Port, R.L., A.G. Romano, J.E. Steinmetz, A.A. Mikhail, and M.M. Patterson. 1986. Retention and acquisition of classical trace conditioned responses by rabbits with hippocampal lesions. Behav. Neurosci. 100: 745-752.

Prokasy, W.F. and J.D. Papsdorf. 1965. Effects of increasing the interstimulus interval during classical conditioning of the albino rabbit. J. Comp. Physiol. Psychol. 60: 249-252.

Steinmetz, J.E. 1990. Classical nictitating membrane conditioning in rabbits with varying interstimulus intervals and direct activation of cerebellar mossy fibers as CS. Behav. Brain Res. 38: 97-108.

Steinmetz, J.E., D.G. Lavond, and R.F. Thompson. 1989. Classical conditioning in rabbits using pontine nucleus stimulation as a conditioned stimulus and inferior olive stimulation as an unconditioned stimulus. Synapse 3: $225-233$.

Thompson, R.F. and D.J. Krupa. 1994. Organization of memory traces in the mammalian brain. Annu. Rev. Neurosci. 17: 519-549.

Yeo, C.H. 1991. Cerebellum and classical conditioning of motor responses. Activity driven CNS changes in learning and development. Ann. N.Y. Acad. Sci. 627: 292-304.

Received March 25, 1997; accepted in revised form May 8, 1997.

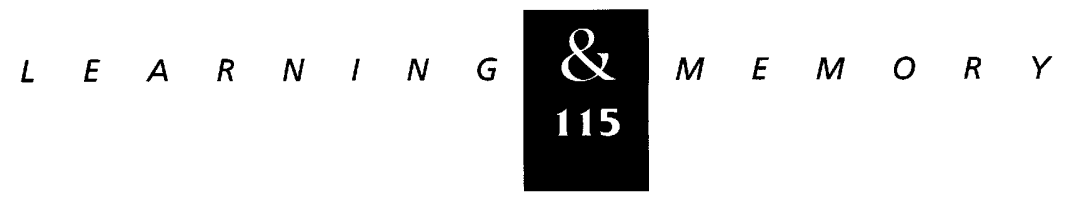




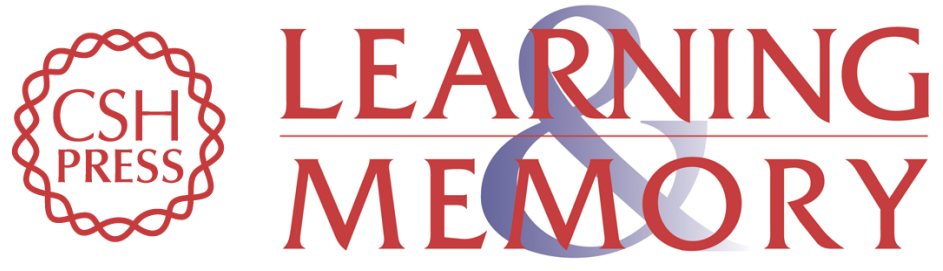

Effect of varying the intensity and train frequency of forelimb and cerebellar mossy fiber conditioned stimuli on the latency of conditioned eye-blink responses in decerebrate ferrets.

P Svensson, M Ivarsson and G Hesslow

Learn. Mem. 1997, 4:

Access the most recent version at doi:10.1101//m.4.1.105

References This article cites 27 articles, 2 of which can be accessed free at: http://learnmem.cshlp.org/content/4/1/105.full.html\#ref-list-1

License

Email Alerting

Receive free email alerts when new articles cite this article - sign up in the box at the Service top right corner of the article or click here. 